\title{
An Emblematic Defibrillator Problem
}

\author{
Robert Hauser ${ }^{1}$ and Jay Sengupta ${ }^{2}$ \\ ${ }^{1}$ Minneapolis Heart Institute Foundation \\ ${ }^{2}$ Minneapolis Heart Institute at Abbott Northwestern Hospital
}

February 1, 2021

\section{JCE-21-0127}

\section{An Emblematic Defibrillator Problem}

Robert G. Hauser MD FACC FHRS and Jay D. Sengupta MD FACC FHRS

Minneapolis Heart Institute Foundation, 920 East 28th Street, Minneapolis, MN 55407, USA

\section{Corresponding Author:}

Robert G. Hauser MD FHRS

Email: rhauser747@gmail.com

Permanent address:

1622 Dusty Drive

Long Lake, MN 55356

Cell: $612-859-5200$

\section{Conflicts of Interest:}

Robert G. Hauser MD FHRS: Cardiac Insight Inc, scientific advisory board

Jay D. Sengupta MD FHRS: None

Word count: 943

Funding: None

Remarkable progress in implantable defibrillator battery technology began in 2008 when Boston Scientific introduced its lithium manganese dioxide battery. This was followed by Abbott in 2010 and Medtronic in 2013 when they began using their versions of the lithium hybrid silver vanadium oxide/carbon monofluoride battery. These novel energy sources have significantly extended the longevities of implantable defibrillators. ${ }^{1}$

However, every battery has finite usable ampere-hour capacity, and anything that excessively drains that capacity will shorten battery life and potentially cause premature and possibly rapid battery depletion. ${ }^{2}$ Manufacturers provide estimates of battery life based on usage profiles that reflect percentage pacing, numbers of pacing sites and delivered or diverted shocks, and use of diagnostic and monitoring features. These estimates no longer apply when a component or design feature malfunctions or misbehaves and draws excessive current from the battery.

Such is the case with certain Emblem subcutaneous implantable cardioverter defibrillators (S-ICD). In this issue of the Journal, $\mathrm{Ip}^{3}$ reports his center's experience with Emblem S-ICD models A209 and A219 (Boston 
Scientific, Inc., St. Paul, MN, USA). In August 2019, the manufacturer notified physicians that a subset of 400 Emblem S-ICDs could exhibit early battery depletion due to excessive current drain caused by a faulty low-voltage capacitor. A year later, Ip found that four of 118 (3.4\%) non-advisoryEmblem S-ICDs followed at his center were showing a sudden and unexpected $>30 \%$ decrease in remaining battery life. Subsequently, in December 2020, Boston Scientific expanded the August 2019 advisory to include all 42,000 pre-August 2019 Emblem S-ICDs manufactured with the old low voltage capacitor, including those in Ip's report. ${ }^{4}$ Other than the need for early pulse generator replacement, no major adverse clinical events have been reported.

On the surface it may appear that this Emblem S-ICD premature battery issue is just another device recall. However, at the same time, in December 2020, Boston Scientific also notified physicians of two additional Emblem problems: 1) the potential for electrical overstress (EOS) due to short circuiting within the pulse generator, ${ }^{5}$ and 2) fracture of the subcutaneous lead-electrode (S-LE). ${ }^{6}$ Unlike the battery problem, these potential defects could compromise therapy and result in death or serious harm.

Emblem EOS failures occurred in six devices manufactured between May 2015 and December 2017 and were caused by moisture ingress due to underfilling of the insulation material that electrically isolates the pins connecting the header to the printed circuit board within the pulse generator. This created a pathway for short circuiting during shock delivery, and resulted in extensive EOS damage to the Emblem's electronics. Our investigation of this issue revealed that five of the six EOS failures occurred between April 2018 and November 2019.

Although no patient was harmed, why did it take a year for Boston Scientific to notify physicians of this potential defect, especially when it is known that this failure mode can be lethal? ${ }^{7}$

The Emblem 3510 S-LE fracture is caused by a fatigue crack that develops proximal to the defibrillation coil and propagates to the distal sensing conductor and then to the high-voltage defibrillation conductors. When both defibrillation

conductors fracture, shock therapy becomes unavailable. As of December 2020, there have been 27 fractures and one death. The death occurred in a patient whose Emblem issued a high impedance alert 12 months after implant and who had radiographic evidence of an electrode body fracture just distal to the proximal sense ring. The patient apparently resisted S-LE replacement. Our search of the Food and Drug Administration's (FDA) online Manufacturers User Facility Device Experience (MAUDE) database revealed that Boston Scientific had evidence of these fractures in early 2018. Why did the manufacturer not notify physicians of this potential problem earlier? Why did it take over a year for it to make recommendations for Emblem lead monitoring and follow-up?

Regrettably, Boston Scientific's handling of these Emblem issues is representative of the cardiac rhythm management industry's decades long history of non-transparency. Patients rightly expect to be informed by their physicians when their pacemaker or defibrillator may have a potentially harmful or life threatening defect. A patient's autonomy to make his or her own health care decisions cannot be preserved if vital product performance information is withheld. Moreover, physicians cannot provide safe post-implant care if manufacturers keep them in the dark.

Pacemaker and implantable defibrillator patients deserve the same consumer protections afforded airline passengers and automobile owners. Maisel ${ }^{8}$ stated it quite well in 2008, when he wrote, "The Latin motto semper fidelis reminds us to remain faithful to certain core principles. To protect the well-being of the recipients of medical devices and to treat them ethically, we must ensure adherence to the principles of informed consent, patient autonomy, and public disclosure of important safety information."

Industry should follow these principles and disclose critical device information promptly and completely. While semi-annual product performance reports contain valuable data, they are not suitable for communicating time-sensitive information. Implanting and following physicians should be notified by email and letter when a critical pacemaker or implantable defibrillator issue arises. Such communications should provide the information that was recommended by the Heart Rhythm Society in 2006, including the advice of an 
independent panel of experts for patient management..$^{9}$ The goals are straightforward: inform our patients, keep them safe, and avoid unnecessary surgical interventions.

\section{References}

1-Hauser RG, Casey SA, Gitter CB, et al. Reliability and longevity of implantable defibrillators. J Interv Card Electrophysiol (2021). https://doi.org/10.1007/s10840-020-00920-w.

2- Boriani G, Merino J, Wright DW, Gadler F, Schaer B, Landolina M. Battery longevity of implantable cardioverter-defibrillators and cardiac resynchronization therapy defibrillators: technical, clinical and economic aspects. An expert review paper from EHRA. Europace 2018; 20: 1882-1897.

3-Ip, J. Premature battery depletion of EMBLEM subcutaneous implantable cardioverter-defibrillators (SICD). J Cardiovasc Electrophysiol 2021;

4- Naughton A. December 2020 EMBLEM S-ICD accelerated battery depletion physician letter. Available at https://www.bostonscientific.com/en-US/pprc/product-advisories.html. Accessed January 25, 2021.

5-Naughton A. December 2020 EMBLEM S-ICD electrical overstress physician letter. Available at https://www.bostonscientific.com/en-US/pprc/product-advisories.html. Accessed January 25, 2021.

6- Naughton A. December 2020 model 3501 physician letter. Available at https://www.bostonscientific.com/en-US/pprc/product-advisories.html. Accessed January 25, 2021.

7- Gornick CC, Hauser RG, Almquist AK, Maron BJ. Unpredictable implantable cardioverter-defibrillator pulse generator failure due to electrical overstress causing sudden death in a young high-risk patient with hypertrophic cardiomyopathy. Heart Rhythm 2004; 2:681-683.

8-Maisel WH. Semper fidelis-Consumer protection for patients with implanted medical devices. N Engl J Med 2008; 358: 985-987.

9-Carlson MD, Wilkoff BL, Maisel WH, et al. Recommendations from the Heart Rhythm Society task force on device performance policies and guidelines. Heart Rhythm 2006; 1251-1273. 